\title{
Análise comparativa do registro médico-pericial do diagnóstico de transtornos mentais de segurados do Instituto Nacional do Seguro Social requerentes de auxílio-doença
}

Comparative analysis of the expertise recordings of mental disorders diagnoses of secured citizens of the Brazilian National Institute of Social Security requiring sickness benefit

Adriana Kelmer Siano', Luiz Cláudio Ribeiro², Mário Sérgio Ribeiro’

\section{RESUMO}

Objetivos: Analisar comparativamente os registros médico-periciais dos diagnósticos de segurados do INSS requerentes de auxílio-doença apresentando transtorno mental. Método: Estudo retrospectivo de registros de perícias iniciais realizadas em agências da Previdência Social de Juiz de Fora, MG, entre julho/2004 e dezembro/2006. Foram realizadas análises bivariadas de acordo com o local de realização da perícia, categoria de perito médico examinador e período da avaliação Resultados: Transtornos depressivos leves ou moderados e transtornos persistentes do humor $(39,6 \%)$ e os transtornos de ansiedade $(34,5 \%)$ - quadros mais leves que não comprometeriam tanto a capacidade laborativa - foram os diagnósticos mais frequentemente registrados. Dentre as comorbidades, transtornos mentais foram mais frequentes $(33,6 \%)$ que quadros clínicos, especialmente na agência Riachuelo, entre peritos concursados após 2005 e no quarto período estudado. A concordância entre o diagnóstico do benefício atual e o do benefício anterior foi baixa, inferior a 50\% na maioria dos casos, mesmo para transtornos mentais graves e com características clínicas mais bem definidas,

\section{Palavras-chave}

Transtornos mentais, incapacidade, Previdência Social.

como as psicoses. A maior taxa de concordância ocorreu com os transtornos por uso de substâncias psicoativas entre peritos credenciados (66,7\%). Conclusão: Este estudo evidencia possíveis falhas no treinamento dos peritos médicos de Juiz de Fora no que se refere ao registro do diagnóstico dos transtornos mentais dos segurados avaliados.

\begin{abstract}
Objectives: Comparative analysis of the expertise recordings of diagnoses of the secured citizens of the Brazilian National Institute of Social Security (INSS) requiring sickness benefit and presenting mental disorders. Method: Retrospective study of the recordings of initial expertise examinations performed in the INSS offices in Juiz de Fora, MG, Brazil, between July/2004 and December/2006.
\end{abstract}

1 Universidade Federal de Juiz de Fora (UFJF), Faculdade de Medicina, Departamento de Clínica Médica, Laboratório de Pesquisas em Personalidade, Álcool e Drogas (Lappda). 2 UFJF, Departamento de Estatística, Instituto de Ciências Exatas, Laboratório de Estudos Estatísticos na Saúde (ICE/LEES). 


\section{Keywords}

Mental disorders, disability, social security.
Bivariate analyses according to the site where the expertise examination was conducted, category of the medical examiner and period of assessment were undertaken. Results: Mild or moderate depressive disorders and persistent mood disorders (39.6\%) and anxiety disorders (34.5\%) - milder conditions that would not impair labor ability - were the most frequently recorded diagnoses. Among comorbidities, mental disorders were more frequent (33.6\%) than clinical ones, especially at the Riachuelo office, involving examiners admitted after 2005 and during the fourth period studied. Concordance rates between the present diagnosis and the previous one were low, lower than 50\% in most cases, even for severe mental disorders with better defined clinical characteristics, such as psychoses. Higher concordance rates were seen for disorders due to the use of psychoactive substances and certified expertise examiners (66.7\%). Conclusion: This study shows possible failures in the training of Juiz de Fora expertise examiners about the recording of the diagnoses of mental disorders of the secured citizens assessed.

\section{INTRODUÇÃO}

Criado em 1990, por meio do Decreto no 99.350', o Instituto Nacional do Seguro Social (INSS) é o atual responsável pela concessão e manutenção dos benefícios previdenciários, dentre os quais se destaca o auxílio-doença. A concessão desse benefício - devido a segurados que, em virtude de algum agravo à saúde, se mostram temporariamente incapacitados para o exercício de suas atividades laborativas sofreu um importante aumento na última década, saltando de 575.742 benefícios concedidos em 1988 para 793.825 em 2001, e daí para 1.288.270 em 2002, chegando a 1.860.695 em $2005^{2}$. O aumento na concessão de benefícios por incapacidade, entretanto, não é exclusividade do Brasil e também vem sendo identificado em outros países ${ }^{3-7}$. Em 2005, começaram a ser efetivadas mudanças institucionais e legais no âmbito do INSS - descritas a seguir -, que se refletiram numa redução inicial de $21,66 \%$ nas concessões de auxíliodoença entre outubro de 2005 e maio de $2006^{8}$.

No processo de concessão do auxílio-doença, tem relevância o trabalho da Perícia Médica Previdenciária. Baseados em normas técnicas, administrativas e legais, os peritos médicos avaliam a incapacidade laborativa dos segurados do INSS - sempre considerando a doença, a idade e a profissão de cada periciado9. Até há poucos anos, por não ter profissionais em número suficiente em seu próprio quadro de pessoal, o INSS também credenciava médicos para a realização de exames periciais. Até novembro de 2000, os laudos emitidos pelos profissionais credenciados eram submetidos à análise dos peritos médicos pertencentes ao quadro de pessoal do INSS para homologação. Entretanto, tal procedimento - que, na prática, já não funcionava como um filtro das decisões dos credenciados e se tornara apenas uma ação burocráti$\mathrm{ca}^{10}$ - foi abolido por meio do Decreto no 3.668 ${ }^{11}$. Cerca de quatro anos mais tarde, a Lei no 10.876 , que criou a carreira de Perícia Médica Previdenciária, determinou a contratação de 3 mil peritos médicos por meio de concursos públicos e fixou em fevereiro de 2006 o prazo final para o descredenciamento dos médicos terceirizados ${ }^{12}$.

Nos anos de 2005 e 2006, a legislação previdenciária relativa à concessão de auxílio-doença também sofreu importantes alterações. Em agosto de 2005, foi instituída a Cobertura Previdenciária Estimada (COPES); tal medida buscou reduzir as longas filas de espera do INSS e determinou que, no ato pericial, os peritos médicos fixassem a Data de Cessação do Benefício (DCB) "considerando a previsibilidade de recuperação da capacidade laborativa"13. Com isso, a DCB passou a substituir a Data de Comprovação da Incapacidade $(\mathrm{DCl})$ nas conclusões periciais, eliminando-se as perícias intermediárias: ao final da avaliação, o segurado não mais recebia a data de sua próxima perícia e só retornaria à Agência da Previdência Social (APS) caso não se sentisse recuperado para voltar ao trabalho. Objetivando a revisão da decisão pericial inicial sobre seu benefício, o segurado poderia requerer apenas um Pedido de Reconsideração (PR) e/ou recorrer à Junta de Recursos da Previdência Social (JRPS) ${ }^{13}$. Em maio de 2006, entretanto, foi instituído o Pedido de Prorrogação (PP), podendo o benefício ser prorrogado quantas vezes fosse constatada a presença de incapacidade laborativa ${ }^{14,15}$.

Durante a avaliação para concessão de auxílio-doença, o perito médico, com base no maior número de dados objetivos, busca elementos de convencimento da incapacidade do segurado para o trabalho ${ }^{16}$. Os transtornos mentais (TM) - que por sua particular subjetividade são considerados uma das mais complexas situações a serem periciadas ${ }^{10,17}$ - destacam-se dentre as três categorias diagnósticas mais frequentemente associadas à incapacidade laborativa, seguindo-se às doenças musculoesqueléticas e cardiovasculares ${ }^{5,18-22}$.

Em 1990, os quadros neuropsiquiátricos foram responsáveis por 10,5\% dos gastos mundiais em decorrência de agravos à saúde, embora representassem apenas 0,3\% das causas de mortalidade ${ }^{23}$. Reportando-se a dados da Organização Mundial de Saúde (OMS) de 2005, Prince et al. ${ }^{24}$ ressaltam que os quadros neuropsiquiátricos responderiam por 31,7\% 
de todos os anos vividos com incapacidade - com destaque para depressão, transtornos por uso de álcool, esquizofrenia, transtorno bipolar do humor e demência. Ainda que a OMS 25 já tenha destacado, há vários anos, o impacto econômico decorrente da perda da produtividade de trabalhadores acometidos por TM', estudos sobre sua relevância na Previdência Social não são encontrados em nosso meio.

O objetivo deste trabalho é realizar uma análise comparativa do registro médico-pericial do diagnóstico dos segurados do INSS - que, em decorrência do requerimento de auxílio-doença, passaram por exame pericial inicial (Ax1) na cidade de Juiz de Fora, Minas Gerais, e receberam como diagnóstico principal o registro de algum TM -, correlacionando-o a três variáveis independentes: a) APS onde o exame pericial foi realizado; b) categoria do perito médico examinador, conforme seu vínculo com o INSS; c) período em que a avaliação foi concluída, de acordo com mudanças nas normas previdenciárias relativas ao auxílio-doença e na categoria de peritos médicos.

\section{MÉTODOS}

Este estudo retrospectivo utilizou um banco de dados fornecido pela Empresa de Tecnologia e Informações da Previdência Social (DATAPREV), incluindo variáveis sociodemográficas, clínicas e previdenciárias relativas a perícias ambulatoriais de Ax1 concluídas com diagnóstico principal no grupo F da CID-10, referente aos transtornos mentais e comportamentais, realizadas nas APS Largo do Riachuelo (APS-R) e São Dimas (APS-SD) da Gerência Executiva do INSS de Juiz de Fora (Gex/JF) entre julho de 2004 e dezembro de 2006, conforme descrito em outros estudos $26-28$.

A APS-R tem seu atendimento direcionado a segurados residentes em Juiz de Fora e, por essa razão, foi considerada a APS típica para efeitos comparativos neste estudo. A APS-SD, por sua vez, além de atender a segurados residentes nesta cidade - em sua grande maioria na Região Norte -, presta atendimento a residentes em 26 cidades vizinhas, de pequeno porte, que fazem parte da Gex/JF e não contam com APS própria².

Considerando-se as mudanças na legislação previdenciária e na categoria de peritos médicos conforme o vínculo com o INSS, o período avaliado foi assim subdividido: 1) de julho de 2004 a julho de 2005, englobando os 13 meses do estudo

1. As estimativas da Carga Global de Doenças em 2000 indicavam que os TM e doenças neurológicas responderiam por 30,8\% de todos os anos vividos com incapacidade.

2. Duas dessas cidades, posteriormente, passaram a pertencer à área de abrangência da APS São João Nepomuceno. Requerentes residentes em outras 12 cidades mineiras pertencentes à área de abrangência de outras APS da Gex/JF foram registrados entre os atendimentos incluídos neste estudo (39 registros). que antecederam a implantação da COPES, envolvendo peritos médicos do quadro de pessoal do INSS concursados antes de 2005 ("concursados antigos"), concursados após 2005

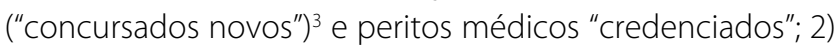
de agosto a novembro de 2005, os quatro primeiros meses após a COPES, incluindo "concursados antigos", "concursados novos" e "credenciados"; 3 ) de dezembro de 2005 a abril de 2006, os cinco meses finais da COPES, antes da instituição do PP, envolvendo "concursados antigos" e "concursados novos"; e 4) de maio de 2006 a dezembro de 2006, englobando os oito meses do estudo após a instituição do PP, com "concursados antigos" e "concursados novos".

Para efeito das comparações aqui realizadas, o primeiro período estudado foi considerado como típico, por ser anterior às mudanças na legislação e no quadro de profissionais médicos do INSS. A tipicidade entre os peritos médicos foi atribuída à categoria dos "concursados antigos", único grupo que realizou avaliações periciais ao longo de todo o período aqui estudado.

Conforme descrito em outro artigo ${ }^{26}$, dos 11.321 registros contidos no banco de dados fornecido pela DATAPREV, foram excluídos 85 que continham informações incorretas sobre algumas datas, como data de nascimento igual a 1/1/2000 e data da entrada do requerimento em 1/1/1900. Para as análises do presente trabalho, os diagnósticos registrados neste banco sob códigos da CID-10 foram recategorizados pelos autores conforme apresentado na Tabela 1.

Tabela 1. Agrupamentos das variáveis estudadas

\begin{tabular}{ll}
\hline \multicolumn{1}{c}{ Variáveis clínicas } & \multicolumn{1}{c}{ Agrupamentos } \\
\hline Diagnóstico & 1) transtornos por uso de substâncias psicoativas ("TUSPA": F10 a \\
psiquiátrico - & F19); 2) "psicoses" (F20 a F29); 3) "transtornos maiores do humor" \\
principal & (transtorno bipolar e depressão grave: F30, F31, F32.2, F32.3, F33.2 \\
& e F33.3); 4) "transtornos menores do humor" (depressão leve ou \\
& moderada e quadros crônicos do humor: demais F32 e F33, F34 a F39); \\
& 5) "transtornos de ansiedade" (F40 a F48); e 6) "outros transtornos \\
& mentais" (demais categorias do grupo F da (ID-10) \\
Diagnóstico & 1) "psiquiátricos"; 2) "cardiovasculares"; 3) "musculoesqueléticos"; e \\
secundário & 4) "outros diagnósticos"
\end{tabular}

Foram realizadas análises descritivas e exploratórias por meio do software SPSS 14.0 (número de série 9656438); nomeadamente, análises bivariadas para avaliar como os registros do diagnóstico dos segurados se distribuem entre as três variáveis que constituem o foco deste estudo: a APS, a categoria do perito médico examinador e o período em que a avaliação foi concluída.

3. Parte dos "concursados novos" iniciou suas atividades na Gex/JF em julho de 2005, pouco antes da instituição da COPES.

4. Apesar de o prazo final estabelecido pela legislação para a conclusão do descredenciamento ter sido fevereiro de 2006, em Juiz de Fora, os Ax1 com registro de TM como diagnóstico principal foram realizados pelos médicos credenciados apenas até novembro de 2005. 
O estudo foi submetido ao Comitê de Ética em Pesquisa da Universidade Federal de Juiz de Fora (CEP/UFJF), recebendo parecer favorável conforme o protocolo de número 041/2007. No desenvolvimento deste trabalho, não houve quaisquer conflitos de interesse.

\section{RESULTADOS}

Quanto ao diagnóstico principal, os "transtornos menores de humor" (39,6\%) - grupamento no qual os autores incluíram os transtornos depressivos leves ou moderados, episódicos ou recorrentes, e os transtornos persistentes do humor - e os "transtornos de ansiedade" (34,5\%) foram os mais frequentemente encontrados em todas as circunstâncias avaliadas (Tabela 2). Entre os Ax1 com diagnóstico secundário, as comorbidades psiquiátricas foram as mais frequentes $(33,6 \%)$, com percentuais mais elevados na APS-R (36,4\%), entre os "concursados novos" $(46,8 \%)$ e no quarto período $(40,0 \%)$. As comorbidades cardiovasculares $(29,8 \%$ do total) foram registradas com menor frequência pelos "concursados novos" e com maior frequência pelos "credenciados": respectivamente, $25,6 \%$ e $35,1 \%$. Nos casos em que foram registradas comorbidades psiquiátricas, o registro de "transtornos de ansiedade" prevaleceu em todas as situações (49,0\%); "TUSPA" e "transtornos maiores do humor" foram mais frequentemente registrados na APS-R $(11,0 \%$ e $8,6 \%$, respectivamente) e pelos "credenciados" (17,6\% e 10,8\%, respectivamente); e as "psicoses" receberam 4,1\% de registros entre os "concursados antigos" (percentual equivalente ao de registros de "psicoses" como diagnóstico principal por essa categoria de peritos).

Entre os Ax1 com TM que possuíam registro de benefício anterior, estes foram devidos, mais frequentemente, a um diagnóstico psiquiátrico, principalmente na APS-R $(62,2 \%)$, entre os "concursados novos" (61,6\%) e no quarto período da pesquisa $(63,9 \%)$; os quadros osteomusculares foram o segundo grupo mais frequente (17,3\%). Enquanto a frequência relativa do registro de doenças cardiovasculares não sofreu grande variação percentual (máximo de 3,3\% entre os

Tabela 2. Diagnóstico dos segurados registrados pela perícia médica (frequência relativa)

\begin{tabular}{|c|c|c|c|c|c|c|c|c|c|c|}
\hline \multirow[t]{2}{*}{ Variável } & \multicolumn{2}{|c|}{$\begin{array}{l}\text { Agências da Previdência Social } \\
\text { (APS) }\end{array}$} & \multicolumn{3}{|c|}{$\begin{array}{l}\text { Categorias de peritos médicos conforme o vínculo } \\
\text { com o INSS }\end{array}$} & \multicolumn{4}{|c|}{$\begin{array}{l}\text { Períodos da pesquisa de acordo com as mudanças na Legislação } \\
\text { Previdenciária e nas categorias de peritos médicos }{ }^{1}\end{array}$} & \multirow[t]{2}{*}{ Total } \\
\hline & APS- $R^{2}$ & APS-SD ${ }^{3}$ & PM-CA & $\mathrm{PM}-\mathrm{CN}^{5}$ & $\mathrm{PM}-\mathrm{Cr}^{6}$ & 10 & $2^{0}$ & $3^{\circ}$ & $4^{0}$ & \\
\hline Diagnóstico principal: & \multicolumn{2}{|c|}{$p<0,001$} & \multicolumn{3}{|c|}{$p<0,001$} & \multicolumn{4}{|c|}{$p<0,001$} & \\
\hline TUSPA7 & 6,1 & 5,9 & 6,3 & 5,5 & 7,6 & 6,6 & 6,0 & 5,2 & 6,0 & 6,0 \\
\hline Psicoses & 4,6 & 3,6 & 4,0 & 4,0 & 7,2 & 5,8 & 4,5 & 3,4 & 3,6 & 4,3 \\
\hline Transt. maiores do humor & 15,1 & 8,4 & 11,3 & 13,4 & 15,2 & 11,7 & 13,6 & 13,4 & 12,5 & 12,6 \\
\hline Transt. menores do humor & 38,1 & 41,9 & 42,9 & 37,3 & 34,6 & 37,7 & 43,1 & 41,3 & 38,8 & 39,6 \\
\hline Transtornos de ansiedade & 33,1 & 36,9 & 33,2 & 36,1 & 32,5 & 35,4 & 30,8 & 34,0 & 35,4 & 34,5 \\
\hline Outros transt. mentais & 2,9 & 3,3 & 2,3 & 3,8 & 2,9 & 2,9 & 2,0 & 2,7 & 3,6 & 3,1 \\
\hline Total de registros (n) & 7.058 & 4.178 & 5.014 & 5.264 & 958 & 2.961 & 1.421 & 2.229 & 4.625 & 11.236 \\
\hline Diagnóstico secundário: & \multicolumn{2}{|c|}{$\mathrm{p}<0,001$} & \multicolumn{3}{|c|}{$p<0,001$} & \multicolumn{4}{|c|}{$p<0,001$} & \\
\hline Psiquiátrico & 36,4 & 28,5 & 24,1 & 46,8 & 25,7 & 24,5 & 37,3 & 35,1 & 40,0 & 33,6 \\
\hline Musculoesqueléticos & 17,7 & 21,0 & 23,7 & 13,5 & 17,7 & 21,1 & 17,8 & 20,3 & 16,3 & 18,9 \\
\hline Cardiovascular & 29,5 & 30,5 & 32,3 & 25,6 & 35,1 & 32,6 & 27,8 & 29,6 & 28,2 & 29,8 \\
\hline Outros diagnósticos & 16,4 & 20,0 & 19,9 & 14,1 & 21,5 & 21,8 & 17,2 & 15,0 & 15,5 & 17,7 \\
\hline Total de registros (n) & 1.755 & 954 & 1.307 & 1.114 & 288 & 857 & 400 & 533 & 901 & 2.709 \\
\hline Diagnóstico secundário & \multicolumn{2}{|c|}{$p=0,5$} & \multicolumn{3}{|c|}{$p=0,08$} & \multicolumn{4}{|c|}{$p=0,5$} & \\
\hline psiquiátrico: TUSPA & 11,0 & 8,5 & 11,1 & 8,6 & 17,6 & 10,7 & 9,4 & 8,0 & 11,4 & 10,2 \\
\hline Psicoses & 2,2 & 2,6 & 4,1 & 1,3 & 1,4 & 4,2 & 2,0 & 1,6 & 1,7 & 2,3 \\
\hline Transt. maiores do humor & 8,6 & 5,9 & 8,6 & 6,9 & 10,8 & 11,2 & 8,1 & 7,0 & 6,1 & 7,8 \\
\hline Transt. menores do humor & 19,9 & 19,1 & 18,1 & 21,3 & 14,9 & 17,8 & 20,1 & 18,2 & 21,4 & 19,7 \\
\hline Transtornos de ansiedade & 47,2 & 53,3 & 47,6 & 50,3 & 45,9 & 45,3 & 50,3 & 54,5 & 47,8 & 49,0 \\
\hline Outros transt. mentais & 11,1 & 10,7 & 10,5 & 11,5 & 9,5 & 10,7 & 10,1 & 10,7 & 11,7 & 11,0 \\
\hline Total de registros (n) & 638 & 272 & 315 & 521 & 74 & 214 & 149 & 187 & 360 & 910 \\
\hline
\end{tabular}

${ }^{1}$ Períodos em meses: $1^{0}=13$ meses; $2^{\circ}=4$ meses; $3^{0}=5$ meses; $4^{0}=8$ meses. ${ }^{2}$ APS Largo do Riachuelo. ${ }^{3}$ APS São Dimas. ${ }^{4}$ Peritos médicos concursados antigos. ${ }^{5}$ Peritos médicos concursados novos. ${ }^{6}$ Peritos médicos credenciados.

${ }^{7}$ Transtornos por uso de substâncias psicoativas. 
dois primeiros períodos), os percentuais relativos às doenças osteomusculares e aos TM sofreram variações mais acentuadas e sempre em sentido contrário. Entre os benefícios anteriores com registro de TM, os diagnósticos mais frequentemente encontrados foram, assim como nos Ax1 avaliados, os "transtornos menores do humor" (37,6\%) e os "transtornos de ansiedade" (35,3\%) nas duas APS, entre as três categorias de peritos e ao longo dos períodos da pesquisa (Tabela 3).

Quando se compara o TM registrado como diagnóstico principal no Ax1 avaliado àquele encontrado no benefício anterior, observa-se que a percentagem de concordância entre os dois registros foi pequena, inferior a $50 \%$ na maioria dos casos (Tabela 4). Para a totalidade dos registros, a maior concordância ocorreu para o grupo diagnóstico de "TUSPA" (48,1\%), seguida de "transtornos do humor", "psicoses", "transtornos deansiedade"e"outros transtornos mentais". A mais alta taxa de concordância ocorreu para os diagnósticos de "TUSPA" entre os "credenciados" (66,7\%) e a menor foi encontrada, também entre os "credenciados", para o grupo das "psicoses" (0\%)

Tabela 3. Diagnóstico principal do benefício anterior (frequência relativa)

\begin{tabular}{|c|c|c|c|c|c|c|c|c|c|c|}
\hline \multirow[t]{2}{*}{ Variável } & \multicolumn{2}{|c|}{$\begin{array}{l}\text { Agências da Previdência Social } \\
\text { (APS) }\end{array}$} & \multicolumn{3}{|c|}{$\begin{array}{l}\text { Categorias de peritos médicos conforme o vínculo } \\
\text { com o INSS }\end{array}$} & \multicolumn{4}{|c|}{$\begin{array}{l}\text { Períodos da pesquisa de acordo com as mudanças na Legislação } \\
\text { Previdenciária e nas categorias de peritos médicos1 }\end{array}$} & \multirow[t]{2}{*}{ Total } \\
\hline & APS-R ${ }^{2}$ & APS-SD ${ }^{3}$ & PM-CA ${ }^{4}$ & PM-CN5 & PM-Cr ${ }^{6}$ & $1^{0}$ & $2^{0}$ & $3^{\circ}$ & $4^{0}$ & \\
\hline Diagnóstico & \multicolumn{2}{|c|}{$p=0,02$} & \multicolumn{3}{|c|}{$p=0,03$} & \multicolumn{4}{|c|}{$p=0,01$} & \\
\hline Psiquiátrico & 62,2 & 55,0 & 58,1 & 61,6 & 49,5 & 50,2 & 51,0 & 58,7 & 63,9 & 59,3 \\
\hline Osteomuscular & 15,8 & 19,6 & 17,2 & 16,3 & 27,3 & 24,1 & 22,5 & 17,6 & 14,2 & 17,3 \\
\hline Cardiovascular & 9,8 & 10,1 & 9,8 & 10,0 & 10,1 & 9,2 & 12,5 & 9,9 & 9,6 & 9,9 \\
\hline Outros diagnósticos & 12,2 & 15,3 & 14,9 & 12,1 & 13,1 & 16,5 & 14,0 & 13,8 & 12,3 & 13,5 \\
\hline Total de registros (n) & 1.148 & 766 & 907 & 908 & 99 & 249 & 200 & 547 & 918 & 1.914 \\
\hline Diagnóstico psiquiátrico & \multicolumn{2}{|c|}{$p=0,1$} & \multicolumn{3}{|c|}{$p=0,2$} & \multicolumn{4}{|c|}{$p<0,001$} & \\
\hline TUSPA $^{7}$ & 7,7 & 6,2 & 6,8 & 7,5 & 6,1 & 4,8 & 5,9 & 5,9 & 8,5 & 7,1 \\
\hline Psicoses & 4,9 & 4,5 & 4,2 & 5,0 & 8,2 & 5,6 & 3,9 & 5,3 & 4,4 & 4,8 \\
\hline Transt. maiores do humor & 15,0 & 10,0 & 11,0 & 15,7 & 6,1 & 10,4 & 6,9 & 6,9 & 18,2 & 13,1 \\
\hline Transt. menores do humor & 37,4 & 38,0 & 37,4 & 38,1 & 34,7 & 33,6 & 43,1 & 42,1 & 35,1 & 37,6 \\
\hline Transtornos de ansiedade & 33,1 & 39,2 & 38,1 & 32,2 & 40,8 & 40,8 & 38,2 & 38,6 & 31,9 & 35,3 \\
\hline Outros transt. mentais & 2,0 & 2,1 & 2,5 & 1,4 & 4,1 & 4,8 & 2,0 & 1,2 & 1,9 & 2,0 \\
\hline Total de registros (n) & 714 & 421 & 527 & 559 & 49 & 125 & 102 & 321 & 587 & 1.135 \\
\hline
\end{tabular}

Períodos em meses: $1^{0}=13$ meses; $2^{0}=4$ meses; $3^{0}=5$ meses; $4^{0}=8$ meses. ${ }^{2}$ APS Largo do Riachuelo. ${ }^{3}$ APS São Dimas. ${ }^{4}$ Peritos médicos concursados antigos. ${ }^{5}$ Peritos médicos concursados novos. ${ }^{6}$ Peritos médicos credenciados. 7 Transtornos por uso de substâncias psicoativas.

Tabela 4. Concordância de diagnósticos registrados no benefício anterior e no $A x 1^{1}$

\begin{tabular}{|c|c|c|c|c|c|c|c|c|c|c|}
\hline \multirow[t]{2}{*}{ Variável } & \multicolumn{2}{|c|}{$\begin{array}{l}\text { Agências da Previdência Social } \\
\qquad \text { - APS }\end{array}$} & \multicolumn{3}{|c|}{$\begin{array}{l}\text { Categorias de Peritos Médicos conforme o vínculo } \\
\text { com o INSS }\end{array}$} & \multicolumn{4}{|c|}{$\begin{array}{l}\text { Períodos da pesquisa de acordo com as mudanças na Legislação } \\
\text { Previdenciária e nas categorias de Peritos Médicos }\end{array}$} & \multirow[t]{2}{*}{ Total } \\
\hline & APS- $R^{3}$ & APS-SD & PM-CA ${ }^{5}$ & PM-CN ${ }^{6}$ & PM-Cr ${ }^{7}$ & 10 & $2^{\circ}$ & $3^{\circ}$ & $4^{0}$ & \\
\hline $\begin{array}{l}\text { Concordância: } \\
\text { TUSPA }^{8}\end{array}$ & $45,5^{9}$ & 53,8 & 55,6 & 40,5 & 66,7 & 33,3 & 33,3 & 47,8 & 52,0 & 48,1 \\
\hline Psicoses & 42,9 & 36,8 & 40,9 & 46,4 & 0 & 28,6 & 50,0 & 35,3 & 46,2 & 40,7 \\
\hline $\begin{array}{l}\text { Transt. maiores do } \\
\text { humor }\end{array}$ & 44,9 & 45,2 & 50,0 & 42,0 & 33,3 & 61,5 & 57,1 & 31,8 & 44,9 & 45,0 \\
\hline $\begin{array}{l}\text { Transt. menores do } \\
\text { humor }\end{array}$ & 41,9 & 50,6 & 52,8 & 39,4 & 29,4 & 45,2 & 47,7 & 45,2 & 44,7 & 45,2 \\
\hline $\begin{array}{l}\text { Transtornos de } \\
\text { ansiedade }\end{array}$ & 36,9 & 34,5 & 36,3 & 35,6 & 35,0 & 49,0 & 43,6 & 28,2 & 35,8 & 35,9 \\
\hline Outros transt. mentais & 21,4 & 44,4 & 30,8 & 25,0 & 50,0 & 33,3 & 50,0 & 25,0 & 27,3 & 30,4 \\
\hline
\end{tabular}

'Exame pericial inicial avaliado por este estudo. ${ }^{2}$ Períodos em meses: $1^{0}=13$ meses; $2^{0}=4$ meses; $30=5$ meses; $4^{0}=8$ meses. ${ }^{3}$ APS Largo do Riachuelo. ${ }^{4}$ APS São Dimas. ${ }^{5}$ Peritos médicos concursados antigos. ${ }^{6}$ Peritos médicos concursados novos. ${ }^{7}$ Peritos médicos credenciados. ${ }^{8}$ Transtornos por uso de substâncias psicoativas. ${ }^{9}$ Os percentuais apresentados referem-se à frequência relativa de grupos diagnósticos concordantes entre linha (Ax1 avaliado) e coluna (benefício anterior), com somatório por coluna. 


\section{DISCUSSÃO}

No presente estudo, mais de $85 \%$ dos periciados receberam um diagnóstico de transtornos do humor ou de ansiedade e apenas 4,3\% foram diagnosticados com "psicoses". Os transtornos do humor e de ansiedade são descritos como os TM mais frequentemente encontrados na população e a depressão é referida como a quarta causa de incapacitação; já a esquizofrenia, um dos TM mais deteriorantes e incapacitantes, tem uma prevalência baixa na população geral ${ }^{29-31}$. Em um estudo com pacientes de uma das regiões sanitárias de Juiz de Fora, os transtornos do humor e os de ansiedade foram também os grupos diagnósticos mais frequentemente registrados, tanto por profissionais da Atenção Básica à Saúde quanto por especialistas do nível secundário de assistência à saúde mental ${ }^{32,33}$. Não foram encontrados na literatura trabalhos brasileiros atuais que indicassem os índices de prevalência nacional dos TM; entretanto, por meio da análise de três estudos, Mari et al. ${ }^{34}$ identificaram que a prevalência de todos os TM na população brasileira variou entre $18,8 \%$ e $29,2 \%$, para um ano, e entre $31,9 \%$ e $41,3 \%$, para toda a vida.

Quando se comparam as frequências dos grupos de TM registrados como diagnóstico principal nos Ax1 com os resultados do estudo de Ribeiro et al. ${ }^{32}$, observa-se que, entre os segurados avaliados, o percentual de transtornos do humor foi mais elevado (52,2\% contra 20,4\%), mostrouse semelhante para "transtornos de ansiedade" e "psicoses" (34,5\% e 4,3\%, respectivamente, contra $36,1 \%$ e $4,9 \%)$ e inferior para o diagnóstico de "TUSPA" (6,0\% contra 10,8\%). Notase que os TM de grupos considerados como quadros mais graves foram registrados mais frequentemente pelos peritos na APS-R (4,6\% de "psicoses" e 15,1\% de "transtornos maiores do humor"), enquanto os registros de "transtornos menores do humor" " "transtornos de ansiedade" foram mais frequentes na APS-SD (41,9\% e 36,9\%, respectivamente). Embora não seja um achado exclusivo deste estudo 4,3,35, a rigor não deveria ser encontrada tão elevada frequência de diagnósticos de "transtornos menores do humor" e "transtornos de ansiedade" entre os requerentes de auxílio-doença, já que, em sua maioria, não seriam tão graves a ponto de interferir incisivamente na capacidade laborativa. A OMS ${ }^{25}$, entretanto, já destacou a importância dos transtornos depressivos unipolares como a principal causa de anos vividos com incapacidade em indivíduos de ambos os sexos na faixa etária de 15 a 44 anos, justamente a fase mais produtiva de sua vida.

$\mathrm{Na}$ avaliação do diagnóstico secundário, nota-se que as comorbidades psiquiátricas foram as mais frequentemente registradas, seguidas de quadros cardiovasculares e osteomusculares. Segundo Andrade et al. ${ }^{36}$, a coocorrência de dois ou mais TM é comum e pode chegar a 30\%, de acordo com estudos populacionais; entre as mulheres seria mais frequente a comorbidade entre depressão e ansiedade. Neste estudo, chama a atenção a presença de grupos considerados mais graves registrados como diagnósticos secundários a outros TM - as "psicoses" (2,3\% no geral; 4,1\% entre os "concursados antigos") e os "transtornos maiores do humor" (7,8\% no geral; $10,8 \%$ entre os "credenciados") - e a maior frequência de "TUSPA" como diagnóstico secundário que como principal (10,2\% e 6,0\%, respectivamente): tais resultados podem dever-se tanto a erros diagnósticos quanto ao desconhecimento dos critérios de hierarquia diagnóstica dos TM por parte dos peritos médicos.

No que diz respeito às comorbidades clínicas, Prince et al..$^{24}$ chamaram a atenção para os TM como fatores de risco para o desenvolvimento de doenças transmissíveis e não transmissíveis e sua possível contribuição a danos acidentais e não acidentais; além disso, muitas patologias clínicas aumentariam o risco de TM ou prolongariam seus episódios. Moussavi et al..$^{37}$ encontraram uma prevalência de depressão significantemente mais elevada entre portadores de doenças clínicas crônicas - como artrite, angina e diabetes -, e a presença de depressão mostrou-se associada à piora da condição clínica desses pacientes.

Quando presentes, a maioria dos benefícios anteriores aos Ax1 avaliados deveu-se a um quadro psiquiátrico. Ao comparar o TM registrado como diagnóstico principal no $\mathrm{Ax} 1$ àquele registrado no benefício anterior, observa-se um elevado índice de mudanças diagnósticas, especialmente entre as categorias "transtornos de ansiedade" e "outros transtornos mentais". Destaca-se, entretanto, a baixa taxa de manutenção do diagnóstico para os grupos de TM mais graves: foi de apenas 40,7\% para as "psicoses" e de 45,0\% para os "transtornos maiores do humor". No caso das "psicoses", que se referem a quadros psiquiátricos com características clínicas mais bem definidas, é interessante destacar que $11,1 \%$ dos segurados tiveram o diagnóstico principal alterado para "transtornos de ansiedade" e 18,5\% para "transtornos menores do humor" no Ax1 (dados não apresentados nos resultados). Ainda que devam ser levadas em consideração as dificuldades relativas à confiabilidade dos diagnósticos psiquiátricos ${ }^{38,39}$ e à diferenciação entre quadros depressivos e ansiosos ${ }^{40}$, tais resultados apontam também para a possibilidade de insuficiência de treinamento dos peritos médicos para avaliação de segurados com TM. A depender de sua formação e de seu conhecimento acerca da psicopatologia e semiologia dos transtornos mentais, o perito médico poderá valorizar certos sintomas relatados pelos segurados e, em consequência, tender a registrar mais frequentemente determinados diagnósticos. Deve-se considerar, ainda, a possibilidade de agravamento do transtorno mental - incluindo a ocorrência de comorbidades psiquiátricas - ou mesmo uma melhor definição diagnóstica ao longo de sua evolução. Também não se pode descartar aqui a hipótese de os peritos médicos estarem transcrevendo no sistema informatizado utilizado pela perícia médica, o Sistema de Administração de 
Benefícios por Incapacidade (SABI), os diagnósticos informados nas declarações dos médicos-assistentes.

\section{CONCLUSÃO}

Os resultados deste estudo evidenciam que os TM mais frequentemente registrados - tanto como diagnóstico principal no Ax1 e no benefício anterior quanto como diagnóstico secundário no Ax 1 - foram quadros considerados mais leves: os "transtornos menores de humor" e os "transtornos de ansiedade". Revelam que as frequências de manutenção dos códigos registrados como diagnóstico principal no Ax1, em relação aos registrados no benefício anterior, foram pequenas, mesmo para quadros com características diagnósticas mais bem definidas, como TUSPA, transtornos maiores do humor e psicoses. Sugerem, por fim, que as diferenças nos registros dos diagnósticos dos TM dos segurados avaliados podem ser fruto de uma falta de formação e/ou de treinamento adequado dos peritos médicos da Gex/JF na área da Psiquiatria.

Ressalta-se que a principal limitação deste trabalho reside no fato de sua amostra restringir-se às avaliações realizadas por peritos médicos na cidade de Juiz de Fora, MG, não sendo possível inferir que seus resultados sejam aplicáveis à perícia médica previdenciária em outras gerências do INSS. Por outro lado, ao abordar um tema que, embora relevante, tem sido pouco pesquisado em nosso país, levantam-se importantes questões que merecem ser elucidadas por estudos futuros mais abrangentes.

Em última instância, além de sugerir a necessidade de melhor qualificação dos peritos médicos para o trabalho com segurados com possíveis transtornos mentais, os resultados deste estudo indicam ainda a relevância de se analisarem, de forma conjunta, possíveis associações entre as conclusões médico-periciais sobre a capacidade laborativa e o perfil diagnóstico dos segurados, suas características sociodemográficas e previdenciárias, considerando-se as diferenças entre os locais de realização das perícias, as mudanças na legislação previdenciária e as alterações das categorias de peritos médicos do INSS - o que deverá ser objeto de futura comunicação.

\section{REFERÊNCIAS}

1. BRASIL. Decreto no 99.350, de 27 de junho de 1990. Cria o Instituto Nacional do Seguro Social - INSS, define sua Estrutura Básica e o Quadro Distributivo de Cargos e Funções do Grupo-Direção e Assessoramento Superiores de suas Unidades Centrais, e dá outras providências.

2. DATAPPREV - Empresa de Tecnologia e Informações da Previdência Social. Base de dados históricos do Anuário Estatístico da Previdência Social, 2007. Disponível em: http://creme. dataprev.gov.br/infologo/inicio.htm. Acessado em: maio 2008.

3. Elders LA, Burdorf A, Ory FG. Ethnic differences in disability risk between Dutch and Turkish scaffolders. J Occup Health. 2004;46(5):391-7.
4. Ford FM, Ford J. Non-attendance for Social Security medical examination: patients who cannot afford to get better? Occup Med (Lond). 2000;50(7):504-7.

5. Moncrieff J, Pomerleau J. Trends in sickness benefits in Great Britain and the contribution of mental disorders.J Public Health Med. 2000;22(1):59-67.

6. Mykletun A, Overland S, Dahl AA, Krokstad S, Bjerkeset O, Glozier N, et al. A populationbased cohort study of the effect of common mental disorders on disability pension awards. Am J Psychiatry. 2006;163(8):1412-8.

7. Quadagno J. Incentives to disability in federal disability insurance and supplemental security income. Clin Orthop Relat Res. 1997;(336):11-7.

8. AgPREV - Agência de Notícias da Previdência Social. Ministro: Nelson Machado fala sobre benefícios por incapacidade na Procuradoria Geral da República. Brasília: Agência de Notícias da Previdência Social; 2006 [atualizada em: 6 set. 2006]. Disponivel em: http://www.mpas.gov. br/agprev/MostraNoticia.asp?ld=25152\&ATVD=1\&xBotao=1. Acessado em: 28 dez. 2006.

9. BRASIL. Manual de Perícia Médica da Previdência Social. Brasília; 2002, p. 118

10. Cechin J, Giambiagi F. 0 aumento das despesas do INSS com o Auxílio-doença. Boletim de Conjuntura do IPEA. Rio de Janeiro: IPEA; 2004, p. 81-90.

11. BRASIL. Decreto n 3.668, de 22 de novembro de 2000. Altera 0 Regulamento da Previdência Social, aprovado pelo Decreto no 3.048, de 6 de maio de 1999.

12. BRASIL. Lei no 10.876, de 2 de junho de 2004. Cria a Carreira de Perícia Médica da Previdência Social, dispõe sobre a remuneração da Carreira de Supervisor Médico-Pericial do Quadro de Pessoal do Instituto Nacional do Seguro Social - INSS e dá outras providências.

13. BRASIL. Orientação Interna INSS/DIRBEN no 130, de 13 de outubro de 2005. Assunto: Cobertura Previdenciária Estimada - Conclusão Médico-Pericial.

14. BRASIL. Orientação Interna INSS/DIRBEN no 138, de 11 de maio de 2006. Dispõe sobre os procedimentos de perícia médica.

15. BRASIL. Orientação Interna INSS/DIRBEN no 164, de 26 de março de 2007. Altera a Orientação Interna INSS/DIRBEN no 138, de 11 de maio de 2006.

16. Melo MPP, Assunção AA. A decisão pericial no âmbito da previdência social. Physis (Rio J). 2003;13(2):343-65

17. Gonzaga P. Perícia médica da previdência social. 4. ed. São Paulo: LTr; 2006

18. Boff BM, Leite DF, Azambuja MI. Morbidade subjacente à concessão de benefício por incapacidade temporária para o trabalho. Rev Saude Publica. 2002;36(3):337-42.

19. Moura AAG, Carvalho EF, Silva NJC. Repercussão das doenças crônicas não transmissíveis na concessão de benefícios pela previdência social. Cien Saude Colet. 2007;12(6):1661-72.

20. Sampaio RF, Silveira AM, Parreira VF, Makino AT, Mateo MM. Análise das aposentadorias por incapacidade permanente entre os trabalhadores da Universidade Federal de Minas Gerais no período de 1966 a 1999. Rev Assoc Med Bras (1992). 2003;49(1):60-6.

21. Silveira DBB, Monteiro MS. Morbidade entre trabalhadores de uma instituição de saúde. Saude Debate. 2004;28(68):206-11.

22. Siano AK, Ribeiro LC, Santiago AE, Souza MAA, Ribeiro MS. Relevância dos transtornos mentais entre as perícias médicas de requerentes de Auxílio-doença na Gerência Executiva do Instituto Nacional do Seguro Social de Juiz de Fora - Minas Gerais. HU Revista. 2008;34(04):235-42.

23. Murray (J, Lopez AD. Global mortality, disability, and the contribution of risk factors: Global Burden of Disease Study. Lancet. 1997;349(9063):1436-42.

24. Prince M, Patel V, Saxena S, Maj M, Maselko J, Phillips MR, et al. No health without mental health. Lancet. 2007;370(9590):859-77.

25. OMS. Relatório sobre a saúde no mundo - 2001: Saúde Mental: nova concepção, nova esperança. Geneva; 2001.

26. Siano AK, Ribeiro LC, Santiago AE, Ribeiro MS. Influência de alterações normativas da Previdência Social sobre o perfil de concessão de auxílio-doença relativo a transtornos mentais. Cien Saude Colet. 2009. Disponivel em: http://www.abrasco.org.br/cienciaesaudecoletiva/artigos/artigo_int.php?id_artigo=2878.

27. Siano AK, Ribeiro LC, Santiago AE, Ribeiro MS. Análise descritiva de exames periciais iniciais de segurados do Instituto Nacional do Seguro Social apresentando transtornos mentais. HU Revista. 2009;35(01):25-34

28. Siano AK. Influência de fatores sociodemográficos, clínicos e institucionais na concessão de benefícios por incapacidade laborativa pelo Setor de Perícias Médicas do INSS em Juiz de Fora - MG em requerimentos de segurados com diagnóstico de transtornos mentais [dissertação de Mestrado]. Juiz de Fora: Universidade Federal de Juiz de Fora; 2009. 
29. Dubovsky SL, Davies R, Duvosky AN. Transtornos do humor. In: Hales RE, Yudofsky SC, eds. Tratado de psiquiatria clínica. 4. ed. Porto Alegre: Artmed; 2006, p. 426-521.

30. Ho B-C, Black DW, Andreasen NC. Esquizofrenia e outros transtornos psicóticos. In: Hales RE, Yudofsky SC, eds. Tratado de psiquiatria clínica. 4. ed. Porto Alegre: Artmed; 2006, p. 370-425.

31. Hollander E, Simeon D. Transtornos de ansiedade. In: Hales RE, Yudofsky SC, eds. Tratado de psiquiatria clínica. 4. ed. Porto Alegre: Artmed; 2006, p. 522-602.

32. Ribeiro MS, Alves MJM, Vieira EMM, Silva PM. Concordância diagnóstica entre profissionais dos níveis primário e secundário na atenção à saúde mental. Revista APS. 2008;11(2):126-35.

33. Ribeiro MS, Alves MJM, Silva PM, Vieira EMM. Comparação da assistência em saúde mental em unidades básicas de saúde com ou sem equipe do Programa de Saúde da Família. Rev Psiquiatr RS. 2009;31(1):40-50.

34. Mari JJ, Jorge MR, Kohn R. Epidemiologia dos transtornos psiquiátricos em adultos. In: Mello MF, Mello AAF, Kohn R, eds. Epidemiologia da saúde mental no Brasil. Porto Alegre: Artmed; 2007, p. 119-41.
35. Scott KM, McGee MA, Wells JE, Oakley Browne MA. Disability in Te Rau Hinengaro: the New Zealand Mental Health Survey. Aust N Z J Psychiatry. 2006;40(10):889-95.

36. Andrade LHSG, Viana MC, Silveira CM. Epidemiologia dos transtornos psiquiátricos na muIher. Rev Psiquiatr Clin. 2006;33:43-54.

37. Moussavi S, Chatterji S, Verdes E, Tandon A, Patel V, Ustun B. Depression, chronic diseases, and decrements in health: results from the World Health Surveys. Lancet. 2007;370(9590):851-8.

38. Caetano R. Causas de baja confiabilidad del diagnostico en psiquiatria: una revision critica. Acta Psiquiatr Psicol Am Lat. 1977;(23):296-306.

39. Kates N, Craven M, Crustolo AM, Nikolaou L, Allen C. Integrating mental health services within primary care. A Canadian program. Gen Hosp Psychiatry. 1997;19(5):324-32.

40. Coutinho ESF, Almeida Filho N, Mari JJ. Fatores de risco para morbidade psiquiátrica menor: resultados de um estudo transversal em três áreas urbanas no Brasil. Rev Psiquiatr Clin (Sao Paulo). 1999;26(5):246-56. 